\title{
Understanding how Social Media Imagery Empowers Caregivers: an Analysis of Microcephaly in Latin America
}

\author{
Vivian Genaro Motti, Niloofar Kalantari \\ Department of Information Sciences and Technology \\ Fairfax, VA \\ vmotti@gmu.edu
}

\begin{abstract}
This paper reports the results of an imagery analysis about Microcephaly in Latin America from the perspective of caregivers. The analysis includes 500 Instagram images associated with the hashtag Microcefalia. Microcephaly affected more than 4,000 babies in Latin America, and the outbreak of the epidemic made Instagram an accessible tool for caregivers to express their thoughts, feelings, raise funds and awareness, exchange information about the condition and find peer support. Most publications are managed by the mother of female patients seeking to celebrate milestones, exchange information, and find social support. The analysis of the contents posted elicits the caregivers' perspectives about microcephaly and clarifies how Instagram empowers them to raise awareness and communicate about the condition. Instagram demonstrated to be low-cost tool to provide support for caregivers pervasively, supporting healthcare practitioners in disseminating medical information. By allowing users to share their individual perspectives and experiences through pictures, images and videos, Instagram has become popular for users promptly seeking for information and support.
\end{abstract}

\section{CCS CONCEPTS}

- Human-centered computing $\rightarrow$ Empirical studies in HCI;

\section{KEYWORDS}

social media analytics, social networks, Instagram, microcephaly

\section{INTRODUCTION}

The epidemics of Zika virus affected 27 American countries, leading to 3037 cases of microcephaly in children born between 2015 and 2017 in Brazil [20], [26]. Patients with microcephaly present multiple impairments thus to be properly treated, these patients need multiple healthcare services, however the services available where microcephaly has high incidence are insufficient to attend patients requiring specialized care. For caregivers of patients with microcephaly, social support is key for exchanging experiences, seeking advice, and information. Web-based solutions and social media facilitate these activities, enabling users to find social support and information of interest and to discuss personal experiences using an inexpensive and accessible tool [22], [14], [15].

Permission to make digital or hard copies of part or all of this work for personal or classroom use is granted without fee provided that copies are not made or distributed for profit or commercial advantage and that copies bear this notice and the full citation on the first page. Copyrights for third-party components of this work must be honored.

For all other uses, contact the owner/author(s).

WOODSTOCK'97, fuly 1997, El Paso, Texas USA

(c) 2016 Copyright held by the owner/author(s).

ACM ISBN 123-4567-24-567/08/06.

https://doi.org/10.475/123_4
While no dedicated application has been created to meet these requirements, caregivers rely on social media channels (such as Twitter and Instagram) to search for medical information and advice, to provide recommendations and connect with peers. For caregivers and parents, social media serves as information source [6]. Its benefits impact patients, caregivers, and practitioners who share information about their medical conditions and treatments. Social support has valuable impact on mental and physical health of users [34], and online groups are successful to decrease depression and increase quality of life [30]. Besides seeking health information online, caregivers are also active users of social media [35]. Despite risks involving privacy and social comparisons [10], social media aids in healthcare enabling users to find support, information and motivation [21]. Thanks to the usage of social media to exchange medical information and provide support [33], social media analytics has become viable for large-scale public health studies [18].

Instagram reached 800 million active users in 2017 [1]. With a mobile and a web application, it allows users to share images, hashtags and descriptions [2]. Instagram stores visual imagery and through follow and search options, it connects peers with common interests. Instagram has the most users in Latin America: over 115 million people [3], the second largest number worldwide [4]. Brazil ranks second in female user share $(58.2 \%)$.

This paper presents an analysis of Instagram imagery. Emergent themes were associated to 500 posts from 2017 and 2018. To analyze the contents discussed about microcephaly and how users adopt Instagram, the questions that guided this study were: 1) What are the profiles of users who use Instagram to communicate about microcephaly? 2) How caregivers use Instagram to communicate about microcephaly? 3) What types of microcephaly-related contents are shared? 4) What are the motivations and rationale for users to discuss microcephaly in Instagram?

To complement the qualitative analysis, after coding all images, we quantified the themes identified. We report the findings of an exploratory analysis of users' behaviors when using Instagram to communicate about microcephaly. We discuss the benefits and drawbacks that social media offers for imagery of medical information from caregivers' perspectives. We focus on caregivers of kids with microcephaly and on how they express themselves using Instagram. We discuss the methods, implications and risks related to the patients' privacy when sharing information. We found that: (1) users who communicate about microcephaly are female (93\%), young adults, mothers of patients; (2) most posts include a photo of a patient in daily activities for documentation and celebration; (3) the post sentiment is positive, to raise awareness about the condition and provide support; and (4) users are unconcerned about privacy and use Instagram to share information or fund raising. 


\section{RELATED WORK}

Social media has been used for medical concerns [18], focusing on recruitment [27], behavior change [16], raising awareness [23] and peer support [36]. Instagram was explored to incentivize physical activity and nutrition habits [11].

Social media analytics was used to study the Zika virus epidemics. With a high-level approach, Zika virus tweets from a 3-month period in 2017 were analyzed, identifying users' locations, profiles, and topics discussed. The discussion began in America (South and North) then spread globally. Centers for Disease Control and the World Health Organization stood out as active participants leading the discussions. Topic modeling and clustering were used to identify key topics, their associations and spatiotemporal patterns. Key topics included pregnancy and abortion. Concerning Instagram imagery, prior analyses focused on information dissemination [31] and comparison between Pinterest and Instagram for communicating about Zika [17].

To study the usage of social media among users concerned about mental health, Instagram imagery was analyzed, showing affordances of self-disclosure, expression of emotional distress and call for help [24]. The disclosure of distress by users was assessed, focusing on miscarriage [7] and the rationale that led users to share information. Interviews and thematic analysis aided to understand users' behaviors. Such studies improve the design of privacy-enhancing technologies for social media, clarifying privacy concerns, but they target at patients and not caregivers. Prior work explored the messages posted on Twitter regarding the concerns and topics of users interested in autism spectrum disorder [9]. The appropriation of Instagram for social support was analyzed concerning cancer among young adults [36], employing text analysis in posts tagged with \#youngadultcancer. Individuals who were survivors fostered higher engagement (based on the number of likes); also the messages exchanged had an overall positive tone and the organizations involved employed emotional contents.

Instagram was studied for information sharing on eating habits, identifying that users seek social support to acquire and provide information about nutrition [12]. To promote weight loss for college students, social networks aided users to hold themselves accountable and to provide peer motivation [25]. For behavior change and smoking cessation [16], social media should be more employed since the target population has regular access to Facebook, Twitter, Pinterest and Instagram, and online interaction and connectedness increase the self-efficacy [29].

Despite several studies on online communities for health [18], the affordances of social media for caregivers is unclear. Prior work assessed social support in online communities to educate parents of kids with special needs in the United States [14]. Parents find peer support in social media, and Facebook is used by most female users aged 18-29 years regardless of racial and socioeconomic lines. These findings are aligned with the theory of online social support [22], [15] which proposes that support in social media helps caregivers of relatives with health concerns [14]. Understanding how social media empowers users to communicate about medical conditions can improve services and aid to derive guidelines for novel systems.

\section{METHODOLOGY}

We collected 500 posts with the hashtag microcefalia, twice to prevent seasonal biases. The posts include jpg images, descriptions and hashtags. The contents are publicly available, and no contact with subjects was made (except for authorization to use images). The Institutional Review Board (IRB) considered the protocol of the study as exempt. To preserve users' privacy, personally identifiable information is removed, pictures are cropped and obfuscated. The hashtags includes 824 different words, acronyms or expressions, mostly in Portuguese. The contents were published from November 2017 to August 2018. In January 2018, Instagram listed 16,115 posts tagged with \#microcefalia (the translation of microcephaly in Portuguese and Spanish). Microcefalia was used since the Latin American regions with highest incidence of microcephaly speak Portuguese and Spanish. We used a mixed-method data analysis, combining qualitative and quantitative methods. Hashtags and descriptions were analyzed based on their nature. Emergent themes aided manual classification. Sentiment metrics were applied to identify valence and tone (positive, neutral or negative). Automated tools are suited for quantitative analysis of data. For a fine-grained analysis, qualitative aspects, and semantic interpretation, manual approaches (such as: coding) are well-suited. The methods used were based on an exploratory analysis with Keyhole on a subset of images to identify high level themes, inspired by prior work [10]. 10 unrelated posts were discarded; 490 images remained for analysis.

Human coding was used to extract semantically meaningful and contextually relevant themes (as in [24]). The codes were defined with a bottom-up approach after an initial image analysis. To facilitate the interpretation of results, we created a code book. The codes were: individuals, events, treatments, and sentiments. Individuals were classified per role (caregiver, patient, or medical practitioner). Practitioners were identified based on their activities (e.g. therapeutic settings) or uniforms. Images with individuals were analyzed based on their age, gender, and activity. Events were classified by purpose (social, educational or fund raising event). Treatments were classified per nature: speech or physical therapy, objects of the picture, including: preps, medical devices, and assistive technologies (e.g. therapeutic suit). Sentiments were classified per valence (positive, negative, neutral), nature (love, faith, hope), and format (text). For images with quotes, text was considered. We extracted the location where the picture was taken, if informed, and the purpose of activities related to exchange of information.

For consensus, experts in Human-Computer Interaction and Public Health analyzed the posts, coded them, quantified the codes, and discussed the results. Most of the contents were in Portuguese (more than $90 \%$ of the posts were from Brazil, according to Keyhole). For comprehensiveness, the words and expressions were translated to English. For quantitative analysis, we combined results from Keyhole [5] (sociodemographic and spatiotemporal data), and LIWC with Portuguese and Spanish dictionaries [28]. Voyant [32], NVivo Plus [8], and Netlytic [19] were used for qualitative analysis of descriptions and hashtags (frequencies and relationships). For graphic analysis, we created word clouds for hashtags frequency, timelines and trend graphics for posts per time, and word trees for post descriptions, using frequent words (nouns) as keywords for contextualization. 


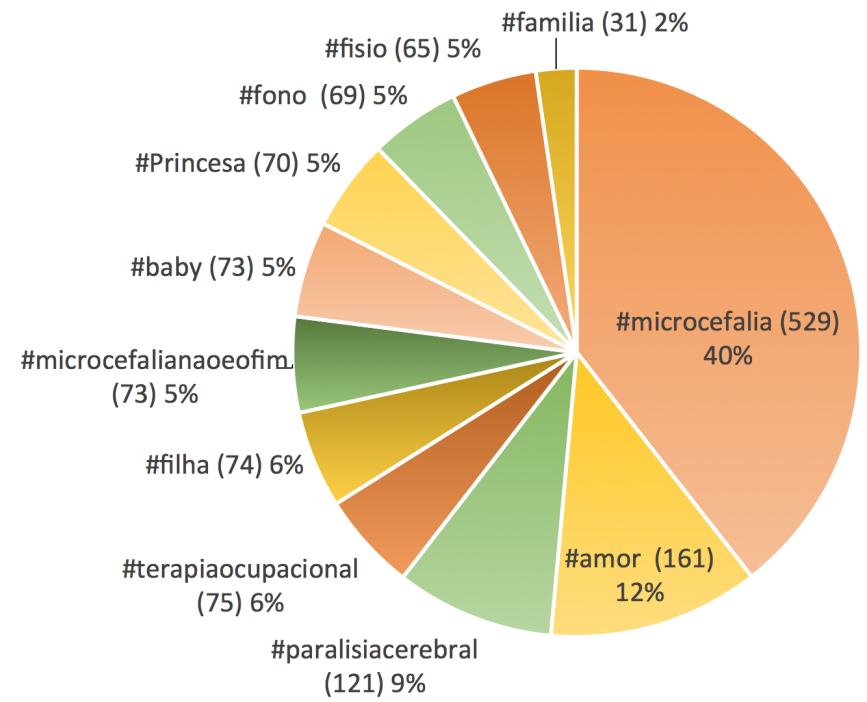

Figure 1: Hashtags extracted from Voyant and nVivo based on descriptions $(n=1341)$ : love (amor) $(12 \%)$, cerebral palsy (paralisia cerebral) (9\%), occupational therapy (terapia ocupacional) (6\%), and daughter (6\%) (filha). The other tags (less than $5 \%$ occurrence) were microcephaly is not the end, baby, princess, speech therapy, physiotherapy, and family

\section{RESULTS}

Using Keyhole we assessed 146 posts related to microcephaly published in two weeks in January 2018 by 57 users. The top posts regarding engagement (averaging 2742 likes) were published by caregivers (a father or a mother). The top posts aimed at raising awareness about the condition and funds for a treatment. Common hashtags (besides microcefalia) were related to: aspects of the disease (e.g. cerebral palsy, encephalopathy, disabled, wheelchair), messages of hope and online campaigns (e.g., microcephaly is not the end, winning microcephaly, winning mielomeningocely, riding with bibi), treatments (occupational therapy, speech therapy, physical therapy, stimulation), and persons (daughter, baby, girls, princess, special kid(s), Sophia, mother of a girl, model) (Figure 1). According to Keyhole, $93.3 \%$ of users were female. The top $30 \mathrm{influ}-$ encers were accounts of parents of patients $(n=7)$ (mostly mothers), patients $(n=10)$, services from commercial or religious organizations $(n=12)$, and an online campaign $(n=1)$. The sentiment of posts was neutral $(53.6 \%)$ or positive (35\%), only $10 \%$ was negative. The sentiment score was 76, showing the proportion of positive posts among all posts classified as non-neutral, suggesting that the interest of the community in building connections leads to a positive tone. The accounts' names (e.g. victory, warrior, angel) and hashtags suggested by Instagram for microcephaly (i.e. microcephaly is not the end, which had more than 7,000 results) corroborates with that, reflecting optimism of the population studied. The top results of user accounts were dedicated accounts created to document the patients' lives, activities and conditions. This is reflected in the names given to the accounts, e.g.: the day of Emily, the world of Lis, Warrior Luana Victory, Leticia wins Microcephaly. Top results of institutional accounts represented foundations, community groups and nonprofit organizations (e.g. Miracle Foundation, Parents of Angels from Bahia, and Embracing Microcephaly). On average, the datasets had 12 hashtags per post $(\max =35)$.

Seven categories emerged from the analysis:

Medical Conditions (comorbidities): encephalopathy, holoprocephaly, myelomeningocele (mielo), cerebral palsy, non-progressive congenital encephalopathy (ECNP), congenital syndrome, delay (language and development), Zika, Congenital Zika Syndrome, Dengue, Malaria, deficit of attention and hyperactivity (TDAH), blindness.

Therapies: speech therapy, physiotherapy, occupational therapy, physical education, hydrotherapy, massotherapy, stimulation.

Support: mother (mom), daughter, father, family, friends, kids, cousins, twins.

Names: Ester, Nicole, Bibi, Malu, Nanda, Maria, Mafe, Lucas, Sophia, Vitoria, Yan, Evely, MicroNanda.

Location: Rio de Janeiro (RJ), Sao Paulo (SP), Bogota, Colombia, Minas (MG), Paraiba (PB), Juiz de Fora, Natal, Tijuca, Gavea;

Campaigns: donation, inclusion, flowered week, draw, raffle, embroidery, crochet, weaving, help, I help, Racism, art, diagnosis is not a destiny, microcephaly is not the end.

Sentiment: love, happy, fun, union, good, faith in life, strength, smile, good day, (only) good news, network of love, our miracle, sweet, thank you, he breathes he inspires me.

To characterize users, the pictures were coded per individual's role, age, gender and number of people. Most pictures include the patient $(n=324)$, a mother $(n=53)$, and a father $(n=28)$. Other persons depicted were family members $(n=7)$, brothers $(n=1)$, cousins or grandmother. Most patients were alone, with the mother or a therapist (identified based on the settings). Most pictures documented daily life. Most patients were children $(n=193)$ or babies $(n=107)$. Seven pictures had a teenager with microcephaly, and five adults (not related to the recent outbreak of Zika virus thus). Most patients were female $(n=222)$, and a minority was male $(n=96)$. A higher proportion of female patients and caregivers was expected, given that female patients present a higher incidence of microcephaly (58\%) [13], besides being majority among Instagram users (58\%) [4]. The proportion of female patients reported by their mothers in the analysis is much higher in the contents analyzed (more than double) when compared with male patients ( $28 \%$ only) for both datasets. Most images included one person $(\mathrm{n}=122)$, two individuals $(n=40)$ or three $(n=8)$. One picture had four persons.

Most posts were from Brazil (92.6\%), as the hashtags in Portuguese indicate, and Keyhole confirmed. Thirty four posts were in Spanish. The word 'Brazilian' stands out as a popular hashtag. Locations mentioned were: Sao Paulo (Vila Mariana), Rio de Janeiro (Gavea), Minas Gerais, Bahia (Salvador), Alagoas and Natal. Despite the Northern states of Brazil concentrating most cases of microcephaly, the Internet access and popularity of social media is higher in Southern states justifying why Sao Paulo and Rio, even with a lower incidence of cases, stand out in the hashtags. Few posts were in Spanish, $10 \%$ only $(n=24)$. The Spanish posts that mentioned a location were from Bogota in Colombia and Maracaibo in Venezuela. Despite the incidence of microcephaly in other Spanish-speaking countries in Latin America, Instagram is widely adopted in Brazil across socio-economic classes, having high online presence. 
Posts refering to faith $(\mathrm{n}=16)$ quoted religious passages. The word 'catholic' stood out among the terms used, as well as 'God', 'bless', 'miracle', and 'angels'. Two images depicted a male patient receiving the visit of a priest, and one picture depicted a female patient at the church, receiving a bless. This finding is expected, given the religious nature of the population. Most posts represent caregivers' views, few posts were published by organizations, institutions, or commercial centers. Publications posted by institutional accounts were related to clinics that offer therapies (e.g. occupational therapy, speech therapy and physiotherapy), a special education school, and academic course offers on neurological development.

\section{CONCLUSION}

This study helps to understand how caregivers use Instagram to discuss microcephaly, and to address their needs for social support, dissemination of information, and celebration of milestones. The results show that caregivers enjoy sharing the daily routines of the patients by publishing pictures that allow them to document the patients' lives and developmental progress. This study provides an in-depth characterization of how Instagram empowers caregivers, as a tool that enables social support, dissemination of information, and fund raising. Despite the privacy risks related to sharing information online, especially related to private events and medical information of kids with cognitive impairments, the users who post online contents seem mostly unconcerned about it, and caregivers enjoy celebrating milestones -sleeping, feeding, brushing teeth, or showering, and assess the progress of the child. Users adopt a positive tone to express their sentiments of gratitude and love.

\section{REFERENCES}

[1] [n. d.]. Instagram Omnicore. https://www.omnicoreagency.com/ instagram-statistics. ([n. d.]). Accessed: 2018-01-15.

[2] [n. d.]. Instagram Social Media Mobile App. https://www.instagram.com/. ([n d.]). Accessed: 2018-01-15.

[3] [n. d.]. Instagram User Base in Latin America. https://www.emarketer.com/ content/instagram-users-in-latin-america-skew-female.. ([n. d.]). Accessed: 2019-01-15.

[4] [n. d.]. Instagram Users in Latin America Skew Female. https://www.emarketer com/content/instagram-users-in-latin-america-skew-female. ([n. d.]). Accessed: 2018-01-15.

[5] [n. d.]. Keyhole: Hashtag Tracking for Twitter, Instagram and Facebook. http: //keyhole.co/. ([n. d.]). Accessed: 2018-01-15.

[6] [n. d.]. PEW Report - Social Media and Parenting. http://www.pewinternet.org/ 2015/07/16/parents-and-social-media/. ([n. d.]). Accessed: 2019-01-15.

[7] Nazanin Andalibi. 2017. Self-disclosure and Response Behaviors in Socially Stigmatized Contexts on Social Media: The Case of Miscarriage. In Proceedings of the 2017 CHI Conference Extended Abstracts on Human Factors in Computing Systems. ACM, 248-253.

[8] Patricia Bazeley and Kristi Jackson. 2013. Qualitative data analysis with NVivo Sage Publications Limited.

[9] Adham Beykikhoshk, Ognjen Arandjelović, Dinh Phung, Svetha Venkatesh, and Terry Caelli. 2014. Data-mining Twitter and the Autism Spectrum Disorder: A Pilot Study. In Proceedings of the 2014 IEEE/ACM International Conference on Advances in Social Networks Analysis and Mining (ASONAM '14). IEEE Press, Piscataway, NJ, USA, 349-356. http://dl.acm.org/citation.cfm?id=3191835.3191906

[10] Elise Rose Carrotte, Ivanka Prichard, and Megan Su Cheng Lim. 2017. Fitspiration on Social Media: A Content Analysis of Gendered Images. Journal of medical Internet research 19, 3 (2017).

[11] Elise R Carrotte, Alyce M Vella, and Megan SC Lim. 2015. Predictors of liking three types of health and fitness-related content on social media: a cross-sectional study. Fournal of medical Internet research 17, 8 (2015).

[12] Chia-Fang Chung, Elena Agapie, Jessica Schroeder, Sonali Mishra, James Fogarty, and Sean A. Munson. 2017. When Personal Tracking Becomes Social: Examining the Use of Instagram for Healthy Eating. In Proceedings of the 2017 CHI Conference on Human Factors in Computing Systems (CHI '17). ACM, New York, NY, USA 1674-1687. https://doi.org/10.1145/3025453.3025747
[13] Wanderson Kleber de Oliveira. 2016. Increase in reported prevalence of microcephaly in infants born to women living in areas with confirmed Zika virus transmission during the first trimester of pregnancy, 2015. MMWR. Morbidity and mortality weekly report 65 (2016).

[14] Beth A DeHoff, Lisa K Staten, Rylin Christine Rodgers, and Scott C Denne. 2016. The role of online social support in supporting and educating parents of young children with special health care needs in the United States: a scoping review. fournal of medical Internet research 18, 12 (2016).

[15] Cindy Dietrich. 2010. Online social support: an effective means of mediating stress. Inquiries fournal 2, 02 (2010).

[16] Jennifer C Duke, Heather Hansen, Annice E Kim, Laurel Curry, and Jane Allen. 2014. The use of social media by state tobacco control programs to promote smoking cessation: a cross-sectional study. Journal of medical Internet research 16, 7 (2014).

[17] Isaac Chun-Hai Fung, Elizabeth B Blankenship, M Elizabeth Goff, Lindsay A Mullican, Kwun Cheung Chan, Nitin Saroha, Carmen H Duke, Marina E Eremeeva, King-Wa Fu, and Zion Tsz Ho Tse. 2017. Zika-Virus-Related Photo Sharing on Pinterest and Instagram. Disaster Medicine and Public Health Preparedness (2017), $1-4$.

[18] Venkata Rama Kiran Garimella, Abdulrahman Alfayad, and Ingmar Weber. 2016. Social Media Image Analysis for Public Health. In Proceedings of the 2016 CHI Conference on Human Factors in Computing Systems (CHI '16). ACM, New York, NY, USA, 5543-5547. https://doi.org/10.1145/2858036.2858234

[19] A Gruzd. 2016. Netlytic: Software for automated text and social network analysis. Computer software, Available from: http://Netlytic. org (accessed 25 May 2017) (2016).

[20] Anne Gulland. 2016. Zika virus is a global public health emergency, declares WHO. (2016).

[21] Maged N Kamel Boulos, Dean M Giustini, and Steve Wheeler. 2016. Instagram and WhatsApp in health and healthcare: An overview. Future Internet 8, 3 (2016), 37.

[22] Sheryl Perreault LaCoursiere. 2001. A theory of online social support. Advances in Nursing Science 24, 1 (2001), 60-77.

[23] Philippe Lenoir, Bilel Moulahi, Jérôme Azé, Sandra Bringay, Gregoire Mercier, and François Carbonnel. 2017. Raising Awareness About Cervical Cancer Using Twitter: Content Analysis of the 2015 \#SmearForSmear Campaign. Fournal of medical Internet research 19, 10 (2017).

[24] Lydia Manikonda and Munmun De Choudhury. 2017. Modeling and Understanding Visual Attributes of Mental Health Disclosures in Social Media. In Proceedings of the 2017 CHI Conference on Human Factors in Computing Systems (CHI '17). ACM, New York, NY, USA, 170-181. https://doi.org/10.1145/3025453.3025932

[25] Gina Merchant, Nadir Weibel, Laura Pina, William G Griswold, James H Fowler, Guadalupe X Ayala, Linda C Gallo, James Hollan, and Kevin Patrick. 2017. Faceto-Face and Online Networks: College StudentsâĂŹ Experiences in a Weight-Loss Trial. fournal of health communication 22, 1 (2017), 75-83.

[26] Health Ministry. 2018. Integrated Monitoring about changes in the growth and development related to Zika Virus Infection and other etiological infections until the Epidemiological Week 48 of 2017. (2018).

[27] Kimberly M Nelson, Jaime J Ramirez, and Michael P Carey. 2017. Developing Online Recruitment and Retention Methods for HIV Prevention Research Among Adolescent Males Who Are Interested in Sex with Males: Interviews with Adolescent Males. Journal of medical Internet research 19, 12 (2017).

[28] James W Pennebaker, Martha E Francis, and Roger J Booth. 2001. Linguistic inquiry and word count: LIWC 2001. Mahway: Lawrence Erlbaum Associates 71, 2001 (2001), 2001.

[29] Joe Phua. 2013. Participating in Health Issue-Specific Social Networking Sites to Quit Smoking: How Does Online Social Interconnectedness Influence Smoking Cessation Self-Efficacy? Journal of Communication 63, 5 (2013), 933-952.

[30] Stephen A Rains and Valerie Young. 2009. A meta-analysis of research on formal computer-mediated support groups: Examining group characteristics and health outcomes. Human communication research 35, 3 (2009), 309-336.

[31] EK Seltzer, E Horst-Martz, M Lu, and RM Merchant. 2017. Public sentiment and discourse about Zika virus on Instagram. Public health 150 (2017), 170-175.

[32] Stéfan Sinclair, Geoffrey Rockwell, et al. 2016. Voyant tools. URL: http://voyanttools. org/[September 5, 2016] (2016).

[33] Anthony Stefanidis, Emily Vraga, Georgios Lamprianidis, Jacek Radzikowski, Paul L Delamater, Kathryn H Jacobsen, Dieter Pfoser, Arie Croitoru, and Andrew Crooks. 2017. Zika in Twitter: Temporal Variations of Locations, Actors, and Concepts. FMIR Public Health and Surveillance 3, 2 (2017).

[34] Shelley E Taylor. 2011. Social support: A review. The handbook of health psychology 189 (2011), 214

[35] Farris K Timimi. 2012. Medicine, morality and health care social media. BMC medicine 10, 1 (2012), 83.

[36] Pontus Wärnestål, Petra Svedberg, Susanne Lindberg, and Jens M Nygren. 2017. Effects of Using Child Personas in the Development of a Digital Peer Support Service for Childhood Cancer Survivors. Fournal of Medical Internet Research 19, 5 (2017). 